\title{
A measure of temporal patterns
}

\author{
JAMES N. MacGREGOR \\ University of Victoria, Victoria, British Columbia, Canada
}

\begin{abstract}
The paper proposes a quantification of temporal patterns derived from Garner's (1974) principles of balance and progression. The measure was found to predict subjects' selection of starting points in the perception of 56 patterns reported in the literature. The median correlation between the measure and performance was -.94 , and 49 of the 56 correlations were significant beyond the .05 level. A model of starting-point selection is proposed to account for the relationship, and a simulation based on the model selected starting points with frequencies similar to those of subjects $(r=.89)$. Further derivations from the model are tested against data in the literature.
\end{abstract}

When a binary pattern is repeated continuously with no cues to indicate a starting point, then any element provides as logically "good" a starting point as any other. However, subjects do not perceive all of the logically possible forms of a pattern with equal frequency. There tend, rather, to be "preferred" forms (Garner \& Gottwald, 1967, 1968; Preusser, Garner, \& Gottwald, 1970; Royer \& Garner, 1966, 1970).

To date, no method of quantifying binary patterns can account for such preferences, but certain descriptive principles have been proposed (Garner, 1974; Royer \& Garner, 1970). These are that preferred patterns display "balance" or "progression." A balanced pattern is one that both begins and ends with the longest runs. Progression occurs when run sizes decrease progressively from the start or increase progressively towards the end of the pattern. To illustrate, the 12-element binary pattern 321123 (in which the integers represent successive run sizes) displays a high degree of both balance and progression and should consequently be a "good" form. In contrast, starting the same sequence on the second singleton run produces 123321 , low in both balance and progression. Consequently, it should be a poorer form, one less likely to be perceived.

The present paper proposes a quantification of Garner's principles. As a first step, it may be observed that balance and progression both depend on two factors, the sizes of runs and their relative positions within a pattern. Run size can be measured as the number of elements in a run. Run position seems to depend on the serial location of a run with respect to the start or end of the pattern. For this reason, it is measured here as the "enclosure" of a run, defined as the number of runs from a given run to the closer end run. The end runs therefore have enclosure values of 1 , the second and second last runs, values of 2 , and so on.

The next step is to observe that both balance and progression are maximized when there is the highest possi-

The author's mailing address is: School of Public Administration, University of Victoria, P.O. Box 1700 , Victoria, B.C., Canada V8W 2 Y2. ble negative correlation between the sizes of runs and their degree of enclosure. This inverse relationship is maximized when the longest runs are in the least enclosed positions (balance), and when the run sizes decrease progressively from the start of the pattern to the middle and increase progressively from the middle to the end (progression). A simple measure of this correlation is given by the sum of the cross-products,

$$
\sum_{i=1}^{n} e_{i} \cdot r_{i}
$$

where $e_{i}$ is the enclosure of the $i^{\text {th }}$ run and $r_{i}$ is its size, for a pattern of $n$ runs. Referring to this measure as $E$, then, a value of $E$ can be calculated for each of the possible starting points of a pattern, and the lowest of the resulting values, $E_{\min }$, should identify the pattern with the greatest degree of balance and progression. (Given the direction of the variable $E$, it is, strictly speaking, a measure of temporal pattern "badness," although the term "goodness" will be used, bearing in mind that the lower the value, the better the pattern.)

Table 1 illustrates the calculation of $E$ for each possible starting point of Pattern P (Royer \& Garner, 1966). The columns of the table show from left to right, the eight possible forms of the pattern, the corresponding run sizes, the enclosure values of the runs, the resulting $E$ values, and the number of subjects perceiving each form (from Royer \& Garner, 1966).

Table 1

Run Size, Run Position, E Value, and Frequency of Selection For All Starting Points of an Eight-Item Sequence

\begin{tabular}{|c|c|c|c|c|}
\hline Pattern & $\begin{array}{c}\text { Run Sizes } \\
\text { r(i) }\end{array}$ & $\begin{array}{l}\text { Position } \\
\text { e(i) }\end{array}$ & $\begin{array}{c}\text { E Value } \\
\sum e(i) \cdot r(i)\end{array}$ & $\begin{array}{c}\text { Frequency of } \\
\text { Selection }\end{array}$ \\
\hline OOXXOXXX & 2213 & 1221 & 11 & 52 \\
\hline XXOXXXOO & 2132 & 1221 & 12 & 38 \\
\hline XXXOOXXO & $\begin{array}{llll}3 & 22 & 2 & 1\end{array}$ & 1221 & 12 & 21 \\
\hline OXXXOOXX & 1322 & 1221 & 13 & 9 \\
\hline XXOOXXOX & 222211 & $\begin{array}{lllll}1 & 2 & 3 & 2 & 1\end{array}$ & 15 & 2 \\
\hline XOOXXOXX & 12212 & $\begin{array}{lllll}1 & 2 & 3 & 2 & 1\end{array}$ & 15 & 2 \\
\hline OXXOXXXO & 12131 & 12321 & 15 & 2 \\
\hline XOXXXOOX & $\begin{array}{lllll}1 & 1 & 3 & 2 & 1\end{array}$ & 12321 & 17 & 2 \\
\hline
\end{tabular}


Table 2

Median Correlations Between $\mathbf{E}$ Value and the Frequency of Selection of Temporal Patterns

\begin{tabular}{lccc}
\hline \multicolumn{1}{c}{ Data Source } & $\begin{array}{c}\text { Number of } \\
\text { Patterns }\end{array}$ & $\begin{array}{c}\text { Number of } \\
\text { Elements }\end{array}$ & $\begin{array}{c}\text { Median r of E } \\
\text { With Frequency }\end{array}$ \\
\hline Garner \& Gottwald (1967) & 2 & 5 & -.87 \\
Royer \& Garner (1966) & 16 & 8 & -.85 \\
Royer \& Garner (1970) & 23 & 9 & -.94 \\
Preusser (1972) & 15 & $6-12$ & -.98 \\
\hline
\end{tabular}

The entries in the last two columns illustrate the relationship between $\mathrm{E}$ and performance for this particular pattern. Two aspects of this relationship are worth noting. First, the most preferred pattern is the one with the lowest $\mathrm{E}$ value, as anticipated from Garner's principles. Second, the frequency of selection of the possible forms of the pattern shows an inverse relationship with $\mathrm{E}$. The product-moment correlation between $\mathrm{E}$ and frequency of selection is, in this case, $r=-.86(p<.05)$. This suggests that the selection of good forms is not categorical, but behaves more like a continuous variable which closely follows the distribution of $\mathrm{E}$ values.

This apparent relationship between $\mathrm{E}$ and pattern preferences was further tested using data from Garner and Gottwald (1967), Preusser (1972), and Royer and Garner $(1966,1970)$. The analyses were identical to that described above for the data from Royer and Garner (1966) and Garner and Gottwald (1967). That is, E values were calculated for all possible starting points in a pattern, and the correlation between $E$ and frequency of selection was obtained for each pattern. The Royer and Garner (1966) patterns had eight possible starting points, and this gives the value for $\mathbf{n}$ in the correlation calculations. For Garner and Gottwald (1967), the patterns had five possible starting points. In the case of Preusser (1972) and Royer and Garner (1970), the frequency of selection of run-breaking forms was not reported, being negligibly small. Consequently, the present calculations included only non-runbreaking forms. For Preusser's data, this provided four forms for each pattern. For Royer and Garner (1970), there were 14 patterns with four possible forms and 9 patterns with six forms.

The results of these comparisons are summarized in Table 2 . The second column of the table shows the number of different patterns used in each study, and indicates the number of correlation coefficients calculated for that set of data. The final column gives the median correlation between $E$ and frequency of selection for each set of data.

It is apparent from the table that there is a strong and consistent relationship between $\mathrm{E}$ and pattern preferences. This relationship was tested over 56 patterns, and the median value of the 56 correlations was -.94 . Forty-nine were significantly different from zero $(p<.05)$. The question naturally arises of why such a relationship exists. A partial explanation is proposed below, in the form of a model of temporal pattern selection.

\section{A MODEL OF TEMPORAL PATTERN SELECTION}

A major proposal of the model is that $E$ is a measure of pattern complexity. The model then attempts to account for preferences as a consequence of differential complexity. The model applies to situations in which patterns repeat continuously, and it is assumed that, during pattern acquisition, subjects overtly or covertly attempt to anticipate or track pattern elements. The main components of the model are represented in Figure 1. Two interlocked cycles are proposed, a starting-point-selection cycle and a pattern-learning cycle. The two cycles are considered to operate simultaneously.

The selection cycle consists of four component events-the selection of a starting point, the $E$ value of the sequence from that starting point, the occurrence of overt or covert errors as the subject tries to learn or track the repeating pattern in that form, and "going off track" as a consequence of errors. The relationships between these events are represented by the symbols " + " and " -," indicating direct and inverse relationships, respectively. For example, selection of an initial starting point results in a particular $E$ value, which may be high or low. If it is high, then, by hypothesis, the probability of error will be high; if it is low, the probability of error will be low. If an error occurs, there is some probability of the subject's "going off track," losing his or her place in the sequence (Restle \& Burnside, 1972). If this occurs, the subject will have to pick up the sequence again, which may result in a change of starting point. If this again results in a high $E$ value, then the same cycle of events is likely to be repeated. If it leads to a lowering in $E$, then error probabilities decrease, going off track becomes less likely, and the probability of a starting shift decreases. According to this model, therefore, sequences are more likely to stabilize around low-E-value forms. Notice however, the operation of the learning cycle. This hypothesizes two relationships between errors and trials. As errors continue, trials are continued (by the experimenter), usually to the criterion of zero errors. As trials continue, errors tend to decrease, through learning.

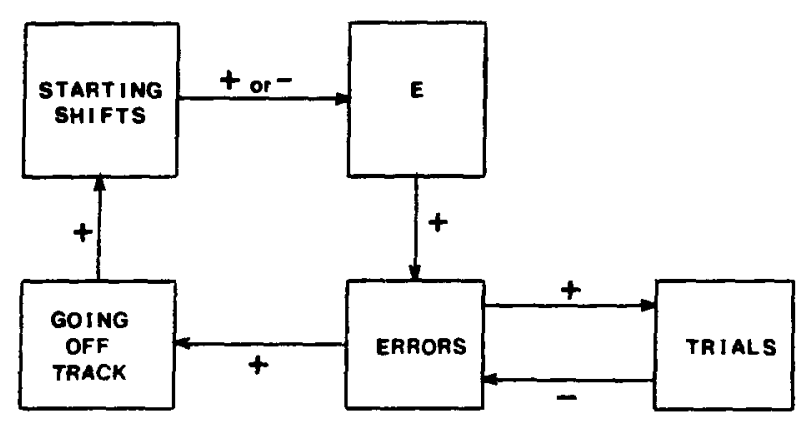

Figure 1. A model of temporal pattern selection. 
The learning cycle makes it possible, therefore, for complex forms to be eventually learned.

As an initial test of this model, a simulation of temporal pattern selection was carried out, in which a computer model performed the following steps. The starting point of a pattern was selected at random, the $E$ value of the pattern determined, and a probability of error assigned to each element proportional to the $E$ value (the constant of proportionality used was a free parameter in the simulations). The occurrence of errors at each element was simulated on the basis of these error probabilities. If no error occurred on the starting element, the program proceeded to the next element in the series. This process continued until an error occurred, at which point a starting point was again selected at random and the entire process was repeated. Following each complete cycle of the sequence, the probability of error was reduced by a constant amount (this simulated the learning cycle, and the constant applied here represented a second free parameter). The program continued until the criterion of two errorless repetitions of the sequence was reached, at which point the sequence was considered to have stabilized and the last starting point was recorded. The frequency that a sequence stabilized around each starting point was the primary data collected.

The simulations employed the 23 unique binary patterns of nine elements. One hundred replications were carried out for each of the 23 patterns, and the frequency of selection of each starting point was obtained. These values were then compared with the percentage of starting points selected by subjects for the same patterns (reported in Royer \& Garner, 1970). Royer and Garner did not report frequency of selection for patterns that break runs, but state that the overall frequency of run-breaking was $1.9 \%$. For comparison with the simulations, I have assigned runbreaking forms a value of 0 throughout.

The goodness of fit of the simulations can be judged by three criteria, the size of the correlation between the predicted and obtained values, the slope of the best-fitting line (predicted value $=1$ ), and the intercept (predicted value $=0$ ). On all three counts, the simulations provided an acceptable fit to the data. The correlation was significantly high $(r=.89, p<.05)$. The obtained slope was .98 , the intercept, .18 . Neither differed significantly from the predicted values.

The results indicate that a process of the kind envisaged by the model leads to the stabilization of the same forms of patterns in approximately the same frequencies as observed in subjects. However, it should be mentioned that the simulations made certain simplifying assumptions that are clearly untenable. Importantly, it was assumed that, for a given starting point, the probability of error was equally distributed across all elements in a pattern. This is wrong on two counts, since errors depend on the position of an element within a run and on the position of a run within a pattern (Jones \& Zamostny, 1975; Restle \& Brown, 1970). Also questionable is the assumption that, when starting shifts occur, the new starting point is selected randomly. It may be more likely that elements that start runs are favored. By not taking these factors into account, the model gives run-breaking forms a better chance to stabilize than should probably be the case, given that subjects virtually never report run-breaking forms (Preusser, 1972; Royer \& Garner, 1970). It is perhaps all the more striking that, with this conservative bias, the simulations closely approximated human performance.

The model makes a number of other predictions about performance, two of which will be explored briefly below. The first is that $E$ should provide a measure of binary pattern complexity. The second pertains to the effects of giving subjects a starting point.

\section{Pattern Complexity}

A major assumption of the model is that $E$ is a measure of pattern complexity. This assumption was tested using response measures of complexity reported in the literature. Table 3 summarizes the results.

From left to right, the columns of the table show the data source, the number of different patterns used, the response measure of complexity employed, and the correlation between $\mathrm{E}$ and complexity. The correlations range from .58 to .99 , and the directional hypothesis that $\mathrm{E}$ correlates with complexity is supported beyond the $95 \%$ level in all seven cases. The lowest correlation is something of an outlier, and Garner and Gottwald (1968) noted that their simplest pattern was often incorrectly described, apparently due to simple carelessness. When this pattern is excluded, the correlation increases to .71 .

\section{The Effects of a Given Starting Point}

In many studies, the effects of a given starting point have been controlled, either by counterbalancing presentation over all starting points (Royer \& Garner, 1966) or by ensuring that subjects "enter" the repeating pattern at a random point (Preusser, 1972; Royer \& Garner 1970). In several studies, however, the effects of a given starting point have been the focus of study (Garner \& Gottwald, 1967, 1968). The present model allows several predictions under these conditions.

Table 3

Correlations of $\mathbf{E}$ With Performance Measures of Complexity

\begin{tabular}{|c|c|c|c|}
\hline Data Source & $\begin{array}{c}\text { Number of } \\
\text { Patterns }\end{array}$ & $\begin{array}{c}\text { Dependent } \\
\text { Variable }\end{array}$ & $\begin{array}{c}\text { Correlation } \\
\text { With E }\end{array}$ \\
\hline $\begin{array}{l}\text { Galanter \& Smith } \\
\text { (1958) }\end{array}$ & 6 & $\begin{array}{r}\text { Median trials } \\
\text { to criterion }\end{array}$ & .98 \\
\hline $\begin{array}{l}\text { Garner \& Gottwald } \\
\text { (1968) }\end{array}$ & 10 & $\begin{array}{l}\text { Errors of } \\
\text { description }\end{array}$ & .58 \\
\hline Preusser (1972) & 15 & $\begin{array}{l}\text { Errors of } \\
\text { reproduction }\end{array}$ & .81 \\
\hline Royer (1967) & 29 & $\begin{array}{l}\text { Maximum rate } \\
\text { of production }\end{array}$ & -.87 \\
\hline $\begin{array}{l}\text { Royer \& Garner } \\
\text { (1966) }\end{array}$ & 19 & $\begin{array}{l}\text { Response point } \\
\text { uncertainty }\end{array}$ & .94 \\
\hline $\begin{array}{l}\text { Scott \& Henninger } \\
\text { (1933) }\end{array}$ & 5 & $\begin{array}{l}\text { Errors to } \\
\text { criterion }\end{array}$ & .99 \\
\hline Vitz (1968) & 20 & $\begin{array}{l}\text { Judged } \\
\text { complexity }\end{array}$ & .90 \\
\hline
\end{tabular}


Consider, first, the probability that subjects will "retain" a given starting point by learning the pattern from the starting point given by the experimenter. For the model, retaining a starting point could mean either that no starting shifts occurred during acquisition or that the subject shifted, then returned to, the initially given starting point. In either case, it follows directly from the model that low $\mathrm{E}$ value forms have a greater chance of being retained than higher $E$ value forms.

Consider, next, the cases in which subjects fail to retain the given starting point. In these cases, the model predicts that lower E-value forms should "attract" responses more frequently than high-E-value forms. That is, the probability that an element will take over as starting point will be negatively correlated with the $E$ value of the resulting pattern.

Garner and Gottwald (1967) report data for two fiveelement patterns that allow a test of these predictions. With respect to retention of starting point, the correlations with $\mathrm{E}$ were -.91 and -.81 . The correlations between $\mathrm{E}$ and "attracting" responses were -.91 and -.79 . All but the last of these correlations were significant beyond the $95 \%$ level. It appears that the capacity of an element to retain or take over the position of starting point varies inversely with $E$ value of the pattern that results.

\section{GENERAL DISCUSSION}

The paper began by proposing a quantification of Garner's (1974) principles of temporal pattern goodness. The proposed measure, E, was found to correlate highly with subjects' pattern preferences. To explain this relationship, a model of starting-point selection was proposed, and simulations based on the model were found to correlate highly with human performance. Central to the model was the proposition that $E$ is primarily a measure of pattern complexity. This proposition was tested using data from seven reported studies, and the median correlation between $\mathrm{E}$ and response measures of complexity was .90 .

The question remains as to why this method of quantifying binary patterns should correlate so consistently with complexity. One possibility is suggested by results in the area of serial pattern learning, which have shown that subpatterns in more enclosed positions are more difficult to learn (Jones \& Zamostny, 1975). If it were also true that the difficulty in learning runs depended directly on their size, then a multiplicative function of run size and run enclosure could provide a measure of total pattern complexity.

Other results in the area of serial pattern learning suggest certain limitations to the present measure, which is based on the assumption that runs provide the basic perceptual unit in the processing of binary patterns. Although, on the one hand, there is considerable support for this as- sumption (Royer \& Garner, 1970; Simon, 1972), there is also evidence that units of a higher order than runs may form, even with binary patterns (Vitz \& Todd, 1969). With more complex patterns, hierarchical forms of encoding appear to be the rule (Jones \& Zamostny, 1975; Restle, 1970; Restle \& Brown, 1970). It seems likely that the present form of the $E$ measure will be useful only in situations in which runs form the important perceptual unit. It is also quite possible that the measure can be generalized to more complex patterns, provided that both the measurement of enclosure and of unit size are based on the relevant units of encoding.

\section{REFERENCES}

Galanter, E. H., \& SMith, W. A. S. (1958). Some experiments on a simple thought problem. American Journal of Psychology, 71, 359-366.

GARNER, W. R. (1974). The processing of information and structure. Potomac, MD: Erlbaum.

GaRner, W. R., \& GotTwald, R. L. (1967). Some perceptual factors in the learning of sequential patterns of binary events. Journal of Verbal Learning and Verbal Behavior, 6, 582-589.

Garner, W. R., \& GotTwald, R. L. (1968). The perception and learning of temporal patterns. Quarterly Journal of Experimental Psychology, 20, 97-109.

Jones, M. R., \& Zamostny, K. P. (1975). Memory and rule structure in the prediction of serial patterns. Journal of Experimental Psychology: Human Learning and Memory, 104, 295-306.

Preusser, D. (1972). The effect of structure and rate on the recognition and description of auditory temporal patterns. Perception \& Psychophysics, 11, 233-240.

Preusser, D., Garner, W. R., \& Gottwald, R. L. (1970). Perceptual organization of two-element temporal patterns as a function of their one-element patterns. American Journal of Psychology, 83, $151-170$.

RESTLE, F. (1970). Theory of serial pattern learning: Structural trees. Psychological Review, 77, 481-495.

RestLE, F., \& BROWN, E. (1970). Serial pattern learning. Journal of Experimental Psychology, 83, 120-125.

Restle, F., \& BURNSIDE, B. L. (1972). Tracking of serial patterns. Journal of Experimental Psychology, 95, 299-307.

ROYER, F. L. (1967). Sequential complexity and motor response rates. Journal of Experimental Psychology, 74, 199-202.

Royer, F. L., \& GARNer, W. R. (1966). Response uncertainty and the perceptual difficulty of auditory temporal patterns. Perception \& Psychophysics, 1. 41-47.

Royer, F. L., \& Garner, W. R. (1970). Perceptual organization of nine-element auditory temporal patterns. Perception \& Psychophysics, 7, 115-120.

Scott, T. C., \& Henninger, L. L. (1933). The relation between length and difficulty in motor learning: A comparison with verbal learning. Journal of Experimental Psychology, 16, 657-678.

Simon, H. A. (1972). Complexity and the representation of patterned sequences of stimuli. Psychological Review, 79, 369-382.

VITZ, P. C. (1968). Information, run structure, and binary pattern complexity. Perception \& Psychophysics, 3, 275-280.

VITZ, P. C., \& TODD, T. C. (1969). A coded-element model of the perceptual processing of sequential stimuli. Psychological Review, 76, 433-449.

(Manuscript received October 15, 1984: revision accepted for publication June 26,1985 .) 